\title{
Isolation and Characterization of S-Enantiomer in Montelukast
}

\author{
Vijaya Lakshmi Maddala ${ }^{1^{*}}$, Kishore Kumar Kakumani ${ }^{2}$ \\ Kameswara Rao Chimalakonda ${ }^{1}$, Srinivasulu Polisetty ${ }^{1}$, P. C. Ray ${ }^{1}$ \\ ${ }^{1}$ Inogent Laboratories Pvt Ltd., IDA-Nacharam, Hyderabad, India \\ ${ }^{2}$ Jawaharlal Nehru Technological University, Hyderabad, India \\ Email: *Vijaya_chem@yahoo.com
}

Received September 22, 2012; revised October 25, 2012; accepted November 3, 2012

\begin{abstract}
A simple and rapid Supercritical Fluid Chromatography (SFC) Method has been developed to isolate and characterize the S-Isomer of Montelukast by using normal phase Amylose based AS-H with $250 \mathrm{~mm} \times 30 \mathrm{~mm}, 5$ microns column using a mobile phase system containing super critical fluid carbon dioxide $\left(\mathrm{CO}_{2}\right)$ and the percentage of 2-Propanol as a mobile phase $(85: 15)$ and detection at $230 \mathrm{~nm}$. The isolated S-Isomer was characterized by using FT-IR, ESI-MS, HPLC and 1H NMR. The purity of isolated S-Isomer is about $98 \%$.
\end{abstract}

Keywords: (S)-Isomer; Montelukast; Supercritical Fluid Chromatography (SFC)

\section{Introduction}

Introduction: Enantiomers of racemic drugs often show different behaviors in pharmacological action and metabolic process. It is not uncommon for one enantiomer to be active while other is toxic in biological systems. The pharmaceutical industry has raised its emphasis on the generation of enantiomerically pure compounds before under taking phamarmacokinetic, metabolic, physiological, and toxicological evaluation in the search for drugs with greater therapeutic benefits and low toxicity $[1,2]$. Nowadays, chiral.

Separations are playing more and more important role for the analysis of single enantiomers in the field of pharmaceutical industry [3]. However, the development of the methods for the quantitative analysis of Chiral compounds and for the assessment of enantiomeric purity is extremely challenging, because the same physical and chemical properties of the two enantiomers make discriminating and separating them very difficulty [4].

Montelukast (sodium salt) is a potent, selective cysteinyl leukotrienes CysLT1 receptor antagonist sold under the trade name Singulair for the treatment of asthma as well as for the symptoms associated enantiomer, with allergic rhinitis. It binds to the human CysLT1 receptor with an IC 50 of less than $5 \mathrm{~nm}$ with no appreciable binding to the CysLT2 receptor. Few HPLC methods are there for the determination of Montelukast sodium were reported in literatures $[5,6]$. The (S)-enantiomer is the

\footnotetext{
"Corresponding author.
}

undesired which can be present as a chiral impurity without any pharmacological and toxicological reports by now. So it is essential to find an effective way to analyze the enantiomers of Montelukast, the chemical structure Montelukast is shown in Figure 1, the chemical structure of (S)-enantiomer is shown in Figure 2.

The aim of this work was is to isolate and characterize the s-isomer of Montelukast. Optimized the chromatographic conditions in terms of mobile phase composition in order to separate and identify the enantiomers of Montelukast, the developed SFC method was used for isolation of (S)-enantiomer in Montelukast.

Supercritical Fluid Chromatography (SFC) is a form of normal phase chromatography that is used for the analysis and purification of low to moderate molecular weight, thermally labile molecules. It can also be used for the separation of chiral compounds. Principles are similar to those of high performance liquid chromatography (HPLC) [7] however SFC typically utilizes carbon dioxide as the mobile phase; therefore the entire chromatographic flow path must be pressurized. Supercritical Fluid Chromatography (SFC) in uses for the separation of chiral molecules, and uses the same columns as standard HPLC systems.

The mobile phase is composed of high pressure liquid or supercritical carbon dioxide, however modifiers are added which can be used to change the chromatography, these are typically alcohols like methanol, ethanol or isopropyl alcohol. Other solvents such as Acetonitrile and chloroform can be used as modifiers. The solvent 
<smiles>CC(C)(O)c1ccccc1CC[C@H](SCC1(CC(=O)O)CC1)c1cccc([C@@H](Cc2ccc3ccc(Cl)cc3n2)SCC2(CC(=O)O)CC2)c1</smiles>

Figure 1. Montelukast R-isomer.<smiles>CC(C)(O)c1ccccc1CC[C@@H](SCC1(CC(=O)O)CC1)c1cccc([C@@H](Cc2ccc3ccc(Cl)cc3n2)SCC2(CC(=O)O)CC2)c1</smiles>

Figure 2. Montelukast S-isomer.

limitations are based on system and column used.

Here, the direct enatioseparation of the undesired enantiomer from an active pharmaceutical ingredient, Montelukast, is isolated by Supercritical fluid chromatography (SFC) using Amylose based AS-H with 250 $\mathrm{mm} \times 30 \mathrm{~mm}, 5$ microns column. The aim of this work was to isolate and characterize the S-enantiomer of Montelukast. Optimized the chromatographic conditions in terms of mobile phase composition in order to separate and identify the enantiomers of Montelukast, the developed SFC method was used for isolation of (S)-enantiomer in Montelukast

\section{Experimental}

\section{Chemicals and Reagents}

Montelukast racemic mixture $( \pm)$ was obtained from the R\&D department of Inogent Laboratories (Hyderabad, India). Chemical structure is presented in Figure 2. HPLC grade 2-Propanal was purchased from Merck (Mumbai, India) and $\mathrm{CO}_{2}$, with a purity of $99.9 \%$ was purchased from MRG enterprises (Hyderabad, India). HPLC grade n-Hexane, Trifluoroaceticacid and Ethanol were purchased from Merck (Mumbai, India).

\section{Isolation by Supercritical Fluid Chromatography (SFC)}

The isolation of S-enantiomer in Montelukast by using SFC 200 System in large scale. The Chromatographic conditions are the total flow was $100 \mathrm{~g} / \mathrm{min}$; the $\%$ of solvent was $15 \%$ isopropanol and the Supercritical fluid is $85 \%$. The automatic back pressure Regulator maintained at 180 , the preparative column was chiral pak AS-H $(250 \times 30 \mathrm{~mm}), 5$ microns. The output signal was monitored by using Gilson detector.

\section{Sample Preparation}

Montelukast racemic mixture $( \pm)$ was prepared with $10 \mathrm{mg} / \mathrm{mL}$ dissolving appropriate amount of the substance in diluent and injected. Collected the S-Isomer fraction distilled and characterized by using spectroscopic techniques.

\section{Characterization of S-Isomer}

\subsection{FT-IR Spectroscopy}

The FT-IR Spectroscopy was recorded in the solid state as $\mathrm{KBr}$ dispersion using Thermo Nicolet 380 FT-IR Spectrophotometer

\subsection{Mass Spectroscopy}

Electrospray ionization mass spectroscopy was performed using a triple quard mass spectrometer. The positive and negative Electrospray MS data was obtained by switching the capillary voltage between $+5000 \mathrm{~V}$ and $-4500 \mathrm{~V}$ respectively.

\subsection{NMR Spectroscopy}

The NMR experiments were performed on Varian 400 $\mathrm{MHz}$. The ${ }^{1} \mathrm{H}$ chemical shift values were reported on the $\mathrm{K}$ scale in ppm.

\subsection{High Performance Liquid Chromatography}

A validated LC method was used for Identification and quantification of S-Isomer of Montelukast. Waters e2695 with 2998 PDA detector with Empower-2 software was used. The chromatographic conditions were Chiral pak AS-H $(250 \times 4.6 \mathrm{~mm}, 5 \mu \mathrm{m})$ column using a mobile phase system containing n-Hexane, ethanol, 2-Propanol, 1,4-dioxane, Trifluoroaceticacid-diethyl amine (65:25: 10:0.3:0.05 v/v). Detection was carried out at $230 \mathrm{~nm}$ and the flow rate $1.0 \mathrm{~mL} / \mathrm{min}$.

\section{Results and Discussion}

Isolation by Supercritical Fluid Chromatography (SFC) Racemic mixture solution of Montelukast and (S)-enantiomer $(10 \mu \mathrm{g} / \mathrm{mL}$ each) prepared in ethanol was used in the method development. To develop a rugged and suitable SFC method for the separation of the two enantiomers, different stationary phases and mobile phases were employed. Initial screening of chiral column was 
carried out by several chiral column suppliers. Various chiral columns, namely Chiralpak AD-H $(250 \times 4.6 \mathrm{~mm})$, chiralpak IC $(250 \times 4.6 \mathrm{~mm})$, chiralcel OD-H $(250 \times 4.6$ $\mathrm{mm})$ of Daicel were employed. All these columns failed to provide selectivity between the two enantiomers by using different possible mobile phases.

It was continued to select the best stationary and mobile phases that would give optimum resolution and selectivity for the two enantiomers. There was an indication of separation on Amylose based AS-H $(250 \times 30$ $\mathrm{mm}, 5 \mu \mathrm{m})$ column using mobile phase consisting of Supercritical carbon dioxide and 2-Propanol. The composition of the mobile phase was optimized to enhance the chromatographic efficiency and resolution between the enantiomers. The result of resolution factor ( $\mathrm{Rs})$ is more than 2.0. Based on the data obtained from the method development and optimization activities, Chiralpak AS-H $(250 \times 4.6 \mathrm{~mm}, 5 \mu \mathrm{m})$ column with mobile phase of Supercritical carbon dioxide and 2-Propanol (85:15\%) was selected forthe final method. The flow rate of the final method was $2.0 \mathrm{~mL} / \mathrm{min}$ with injection volume $20 \mu \mathrm{L}$. The column temperature was $25^{\circ} \mathrm{C}$, and the detection wavelength was $230 \mathrm{~nm}$. Under these conditions, the two enantiomers were separated well and the peak of (S)-enantiomer eluted after the peak of Montelukast. In the optimized method, the typical retention time of Montelukast and (S)-enantiomer were 10.65 and 12.5 min, respectively. Base line separation of Montelukast and (S)-enantiomer were obtained with total run time of $20 \mathrm{~min}$. The separation of an approximately 1:1 (wt/wt) mixture solution (in ethanol) of the two enantiomers in shown in Figure 3. Montelukast peak and S-enantiomer were confirmed with by injecting individually in HPLC. (Figure 4).

Amylose based chiral stationary phase contained five chiral centers per unit. The stereoelectronic interaction

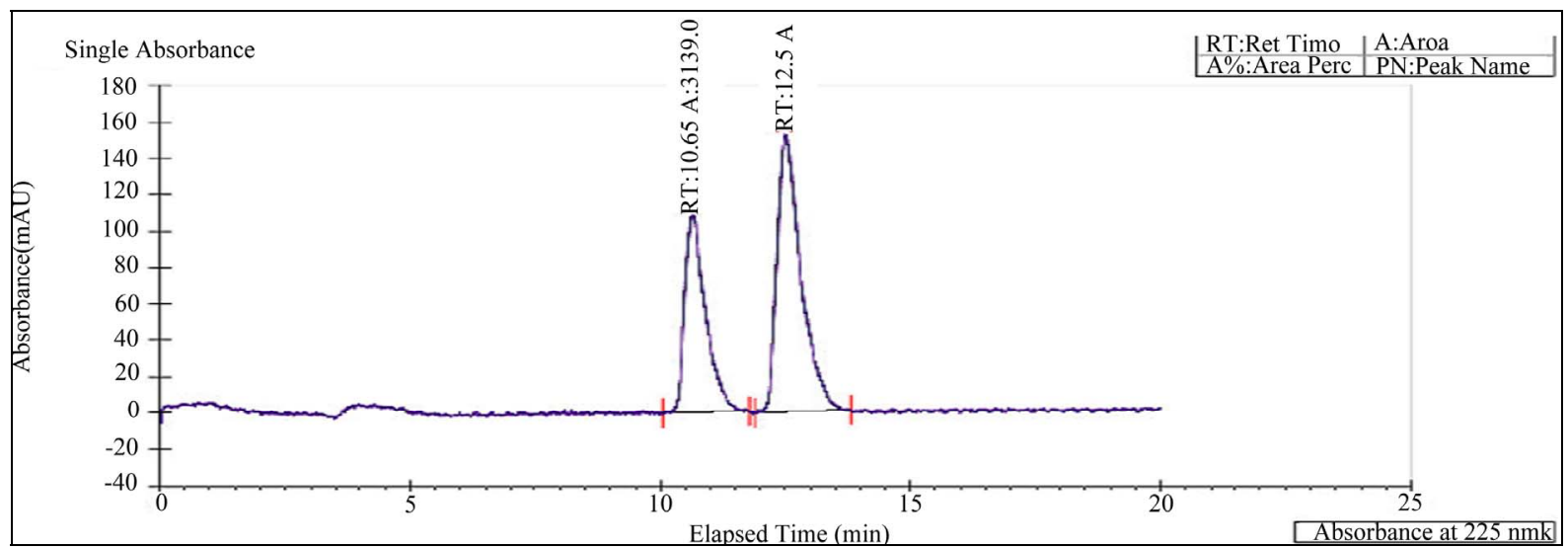

Figure 3. Racemic mixture of montelukast.

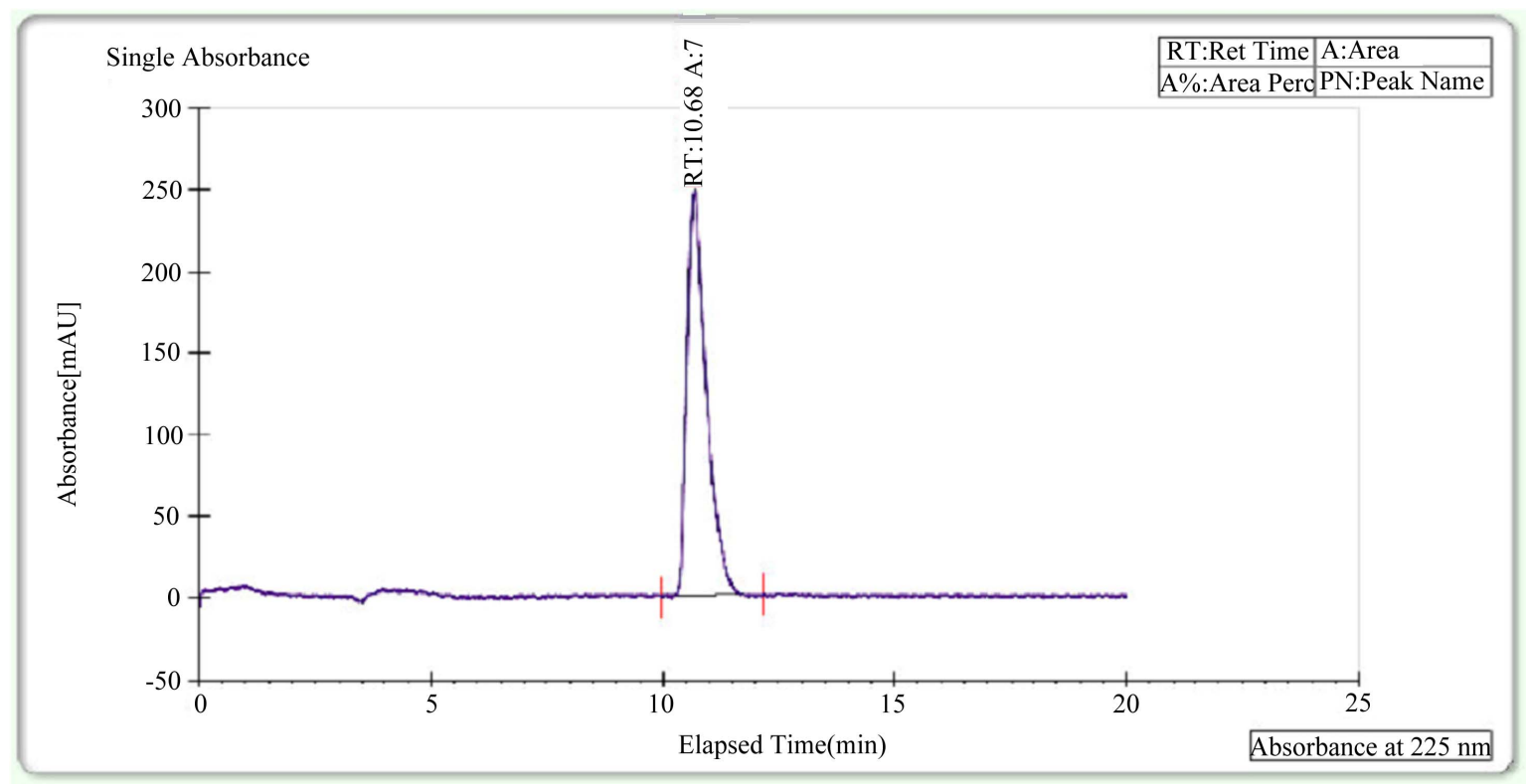

Figure 4. Montelukast sample. 
between the enantiomers and the chiral stationary phase generated enantioselectivity, thus causing significant difference in the migration of the enantiomers inside the column. Having the right amount of 2-Propanol in the mobile phase also played an important role in affecting the steric environment of the chiral cavities or channels of the stationary phase and contributes to enantioselectivity. However, an excessive amount of 2-Propanol was likely to cut down the resolution by taking up chiral centers of the chiral stationary phase or forming hydrogen bonding with enantiomers instead of the hydrogen bonding between the enantiomers and the stationary phase other important interaction between the enantiomers and the stationary phase such as $\pi-\pi$ bonding. Vander walls forces, dipole induced dipole attractions, and steric effects can also achieve better resolution on chiralcel AS-H column.

\section{FT-IR Spectroscopy}

Dispersed about $2 \mathrm{mg}$ of S-Isomer in $300-400 \mathrm{mg}$ of $\mathrm{KBr}$, grind the mixture, spread uniformly on a die and compress in to a thin disk by applying pressure about 800 Mpa. Kept the pellet in $\mathrm{KBr}$ folder in FT-IR spectrophotometer and scanned the spectrum from $4000 \mathrm{~cm}^{-1}$ to 450 $\mathrm{cm}^{-1}$.

Noted the wavelengths and it's confirming the functional groups of S-Isomer and shown at Figure 5.

\section{Mass Spectroscopy}

Analyzed the S-Isomer by using Mass spectroscopy and observed the mass number $(\mathrm{m} / \mathrm{z})$ for S-Isomer and it's confirming the mass number of S-Isomer is $586(\mathrm{~m} /$ $\mathrm{z}$ :negative mode :584) as shown in Figure 6.

\section{NMR Spectroscopy}

S-Isomer sample analyzed 1H NMR using Varian 400 $\mathrm{MHz}$. The no. of protons confirms the structure of SIsomer. The reported $1 \mathrm{H}$ NMR spectrum was shown Figure 7.

\section{High Performance Liquid Chromatography}

A validated LC method was used for Identification and quantification of S-Isomer of Montelukast. Waters e2695 with 2998 PDA detector with Empower-2 software was used. The chromatographic conditions were Chiralpak AS-H $(250 \times 4.6 \mathrm{~mm}, 5 \mu \mathrm{m})$ column using a mobile phase system containing n-Hexane, ethanol, 2-Propanol,1,4dioxane, Trifloroacetic acid and diethylamine (65:25:10: $0.3: 0.05 \mathrm{v} / \mathrm{v})$. Detection was carried out at $230 \mathrm{~nm}$ and the flow rate $1.0 \mathrm{~mL} / \mathrm{min}$.

Both Montelukast and Isolated S-Isomer were prepared in diluent and injected individually and as co-injection. S-Isomer Retention time was confirmed by injected racemic mixture.

\section{Conclusion}

The research paper describes the isolation and characterization of S-Isomer of Montelukast by using Supercritical Fluid Chromatography (SFC). The S-isomer was isolated by using Supercritical Fluid Chromatography

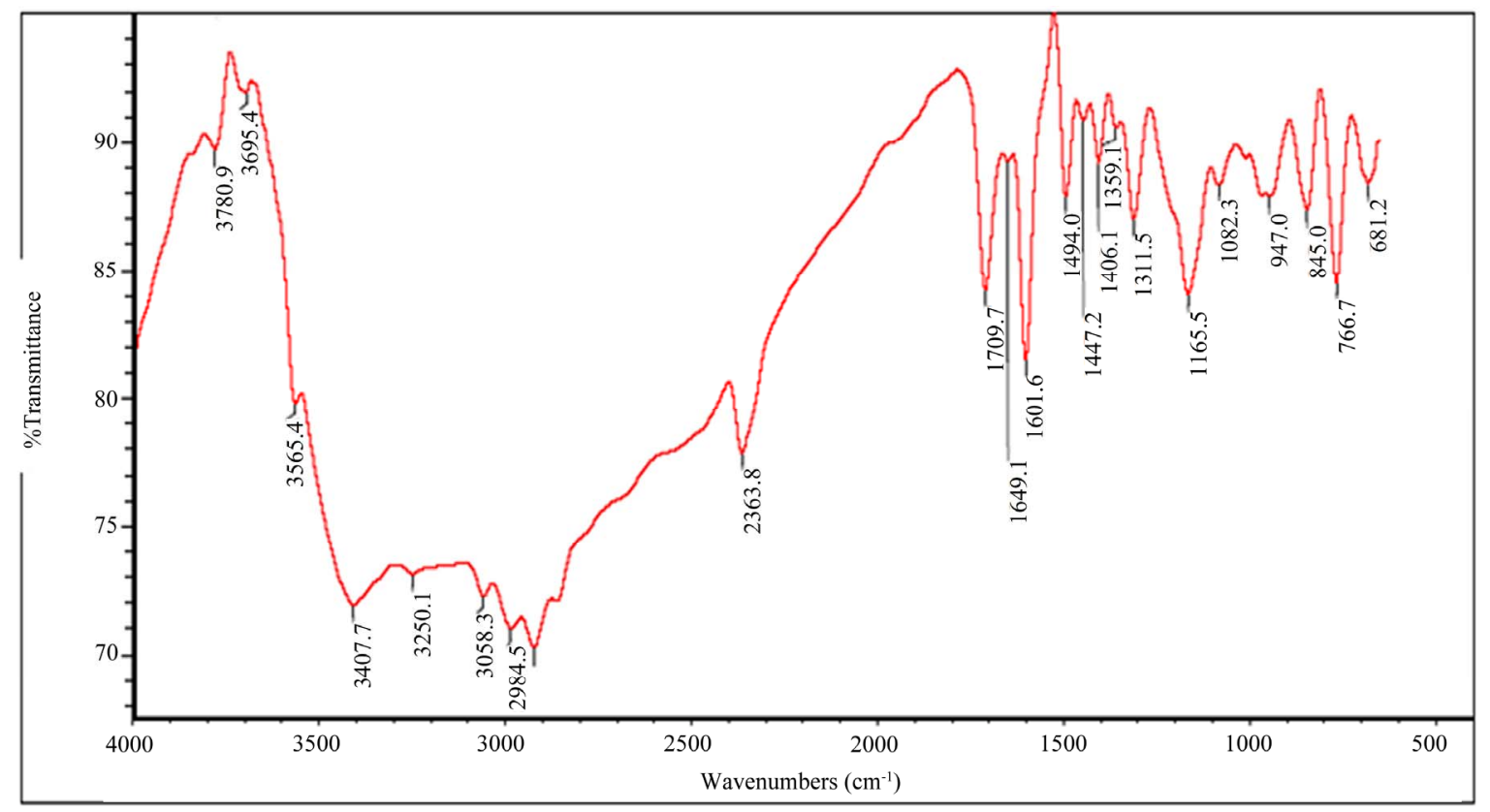

Figure 5. Montelukast S-isomer IR. 


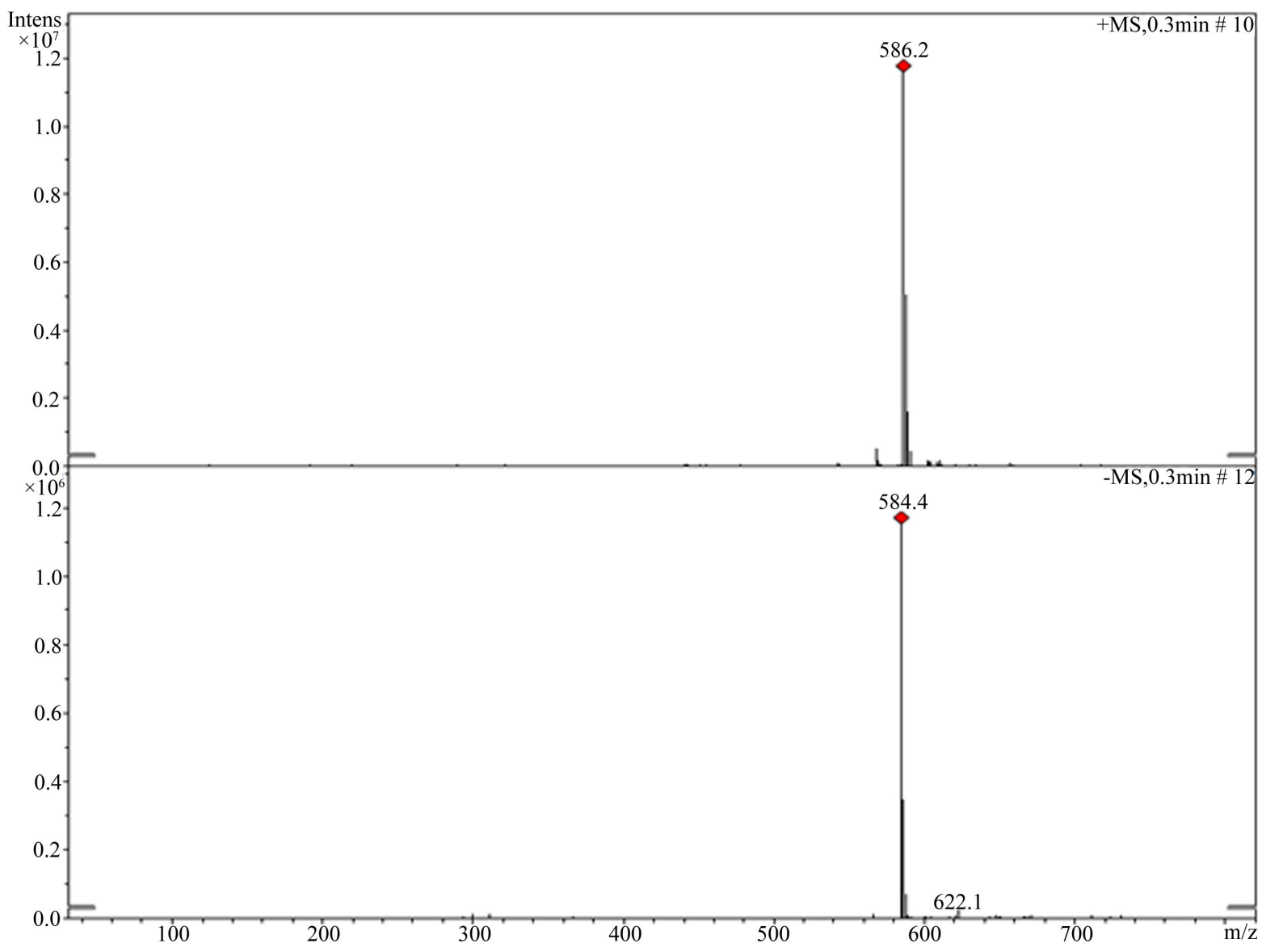

Figure 6. Montelukast S-isomer mass.

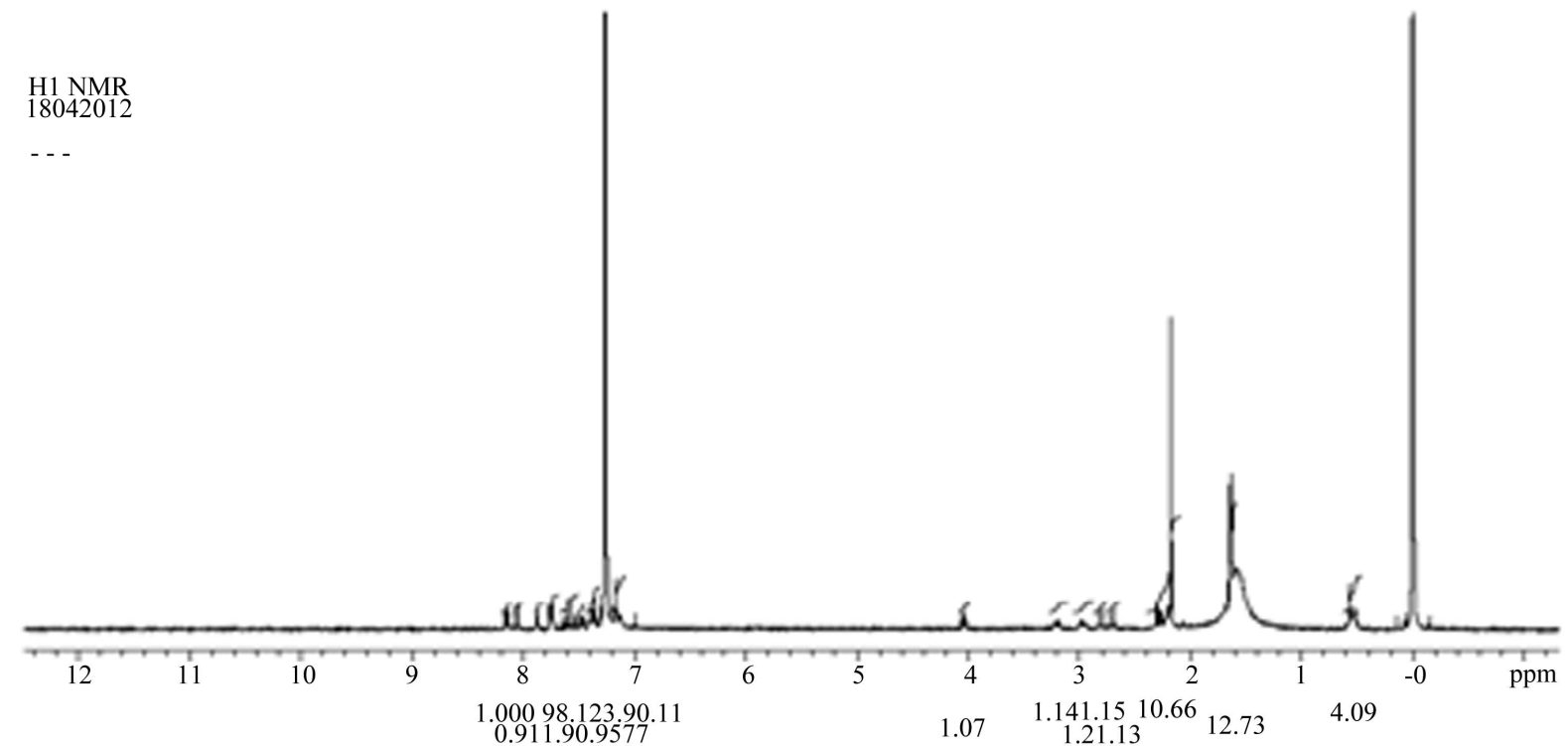

Figure 7. Montelukast S-isomer proton NMR.

(SFC) with Amylose based AS-H $(250 \times 30 \mathrm{~mm}, 5 \mu \mathrm{m})$ column using a mobile phase consisting of Supercritical carbon dioxide and 2-Propanol (85:15). The isolated SIsomer was characterized by using FT-IR, Mass number 
(m/z) m, ${ }^{1} \mathrm{H}$ NMR, and by Chiral HPLC analysis.

\section{Acknowledgements}

The authors would like to acknowledge the management of Inogent Laboratories Private limited, Hyderabad, India.

\section{REFERENCES}

[1] ICH, "Validation of Analytical Procedures: Text and Methodology (Q2(R1)) International Conference on Harmonization," IFPMA, Geneva, 2005.

[2] L. Liu, H. Cheng, J. J. Zhao and J. D. Rogers, "Determination of Montelukast (MK-0476) and S-Enatiomer in Human Plasma by Stereoselective High Performance Liquid Chromatography with Column Switching," Journal of Pharmaceutical and Biomedical Analysis, Vol. 15, No. 5, 1997, pp. 631-638. [Pub Med] doi:10.1016/S0731-7085(96)01894-8

[3] B. Chauhan, S. Rani, M. Nivsarkar and H. Padh, "New Liquid-Liquid Extraction Method for Determination for Montelukast in Small Volume Human Plasma Samples
Using HPLC with Fluorescence Detector," Indian Journal of Pharmaceutical Science, Vol. 68, No. 4, 2006, pp. 517-520. doi:10.4103/0250-474X.27834

[4] N. M. Maier, P. Franco and W. Lindner, "Separation of Enantiomers: Needs Challenges, Perspectives," Journal of Chromatography A, Vol. 906, No. 1-2, 2001, pp. 3-33. doi:10.1016/S0021-9673(00)00532-X

[5] V. Choudari, et al., "Simultaneous Determination of Montelukast Sodium and Levocitrazine Dihydrochloride in Pharmaceutical Preparations by Ratio Derivative Spectroscopy," International Journal of PharmTech Research, Vol. 2, No. 1, 2010, p. 4.

[6] P. Radhakrishnan, D. V. Subbarao, K. V. Surendranath, D. Subrahmanyam and V. Himabindu, "A Validated LC Method for Determination of the Enantiomeric Purity of Montelukast Sodium in Bulk Drug Samples and Pharmaceutical Dosage Forms," Chromatographia, Vol. 68, No. 3-4, 2008, pp. 263-267.

[7] B. S. Sekhon, "Separation of Pharmaceutical Enantiomers Using Supercritical Fluid Technology," International Journal of Pharmtech Research, Vol. 2, No. 2, 2010, pp. 1595-1602. 